\title{
ACTITUD DIAGNÓSTICA Y TERAPÉUTICA EN EL TUMOR GERMINAL BILATERAL. PRESENTACIÓN DE UN CASO Y REVISIÓN DE LA LITERATURA
}

\author{
D. CAÑÍS SÁNCHEZ, G. CONDE SANTOS, N. ALONSO GRACIA, \\ E. DE LEÓN MORALES, O. ARANGO TORO, A. GELABERT MAS
}

Servicio y Cátedra de Urología Hospital del Mar. Univesitat Autónoma de Barcelona. Barcelona.

Actas Urol Esp. 27 (2): 147-151, 2003

\section{RESUMEN}

“ACTITUD DIAGNÓSTICA Y TERAPÉUTICA EN EL TUMOR GERMINAL BILATERAL. PRESENTACIÓN DE UN CASO Y REVISIÓN DE LA LITERATURA"

El tumor germinal bilateral representa entre el 1 y el $4 \%$ de los tumores de testículo y suele plantear al urólogo importantes dificultades en su diagnóstico y tratamiento. Aportamos el caso de un paciente de 30 años, diagnosticado de seminoma con áreas de tumor del saco vitelino en teste izquierdo, que fue tratado con orquiectomía. Once meses después, presentó una segunda neoplasia en el teste contralateral, cuya histología fue de seminoma clásico con carcinoma "in situ" peritumoral, siendo tratado también con orquiectomía.

Se revisa la literatura médica, con especial referencia a los factores de riesgo implicados y al manejo de estos tumores: la detección de carcinoma "in situ" aparece como factor predictivo más potente. Se plantea la realización de biopsia testicular contralateral en casos seleccionados. Aunque la orquiectomía radical sigue siendo el tratamiento de elección, la radioterapia local y la cirugía conservadora representan una alternativa en casos de tumor bilateral.

PALABRAS CLAVE: Tumor germinal de testículo bilateral. Carcinoma in situ. Biopsia contralateral. Cirugía conservadora.

\section{ABSTRACT \\ “DIAGNOSIS AND TERAPEUTIC MANAGEMENT IN GERMINAL BILATERAL TUMORS. A REPORT OF ONE CASE AND LITERATURE REVISION"}

The germinal bilateral tumor represents from 1 to $4 \%$ of the testicle tumors, and it usually turns to be one of the most difficult ones when talking about diagnosis and treatment. We are attaching a 30 years old patient case, with a diagnosis consisting in a seminoma with yolk salc tumor area's from the left testicle, that was treated with orquiectomy. Eleven months later, the patient presented a second neoplasm in the contralateral testicle, whose histology was a classical seminoma with carcinoma "in situ" peritumoral, being also treated with orquiectomy.

Medical literature is revised with emphasis in the involved risk factors and the tecniques used to treat these testis tumor groups: carcinoma "in situ" detection appears to be the strongest predicting factor. Testicular contralateral biopsia is proposed in some select cases. Even though radical orquiectomy is still the treatment to choose, local radiotherapy and consevative surgery are also an option in patients with bilateral tumor.

KEY WORDS: Bilateral germ cell cancer of the testis. Carcinoma "in situ". Contralateral biopsia. Conservative surgery. 
$\mathrm{E}^{1}$ cáncer de testículo representa el proceso maligno más frecuente en varones de 15 a 35 años de edad, siendo ésta una de las neoplasias sólidas con mayores tasas de curación y en la que se cuenta con un mayor arsenal terapéutico.

En España su incidencia se sitúa entre 0,5 y 1,9 casos por cada 100.000 habitantes/año ${ }^{1}$. Si excluimos las neoplasias secundarias, metastásicas y hematológicas, la prevalencia de tumor germinal de testículo (TGT) bilateral se sitúa entre el $1 \%$ y el $4 \%$ y se presentan de forma metacrónica en más del $75 \%$ de los casos según las mayores series publicadas ${ }^{3,9}$. Esta prevalencia sigue incrementándose en las últimas décadas desde la introducción de la quimioterapia sistémica con Cis-platino.

\section{CASO CLÍNICO}

Paciente de 30 años, sin antecedentes familiares ni tóxicos de interés. Entre los antecedentes patológicos destaca ulcus duodenal, laparotomía exploradora por herida de arma blanca, uretritis gonocócica, VHB (+), y varicocelectomía izquierda tipo Palomo a los 18 años.

Consulta por aumento de tamaño del testículo izquierdo de un año de evolución. A la exploración se palpa teste derecho normal e hidrocele izquierdo que dificulta la exploración del testículo. Se solicita una ecografía que demuestra una lesión sólida e hipoecoica de $17 \mathrm{~mm}$ de diámetro mayor localizada en el polo inferior del teste izquierdo (Fig. 1).

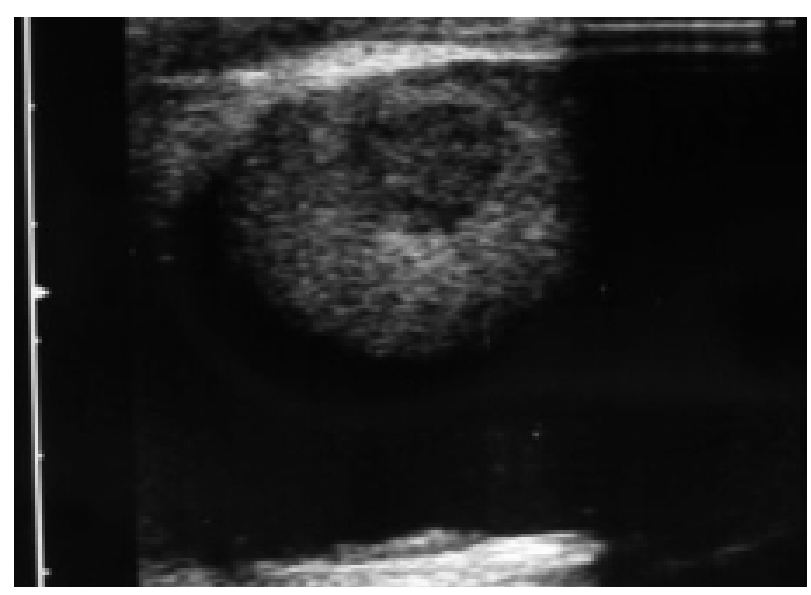

Figura 1. Lesión sólida e hipoecoica de $17 \mathrm{~mm}$ de diámetro mayor en polo inferior de testiculo izquierdo.
Los marcadores tumorales séricos (LDH; $\beta$ HCG; $\alpha$-AFP) fueron estrictamente normales. La radiografía simple de tórax y TC abdómino-pélvico fueron negativos para metástasis.

Se realiza inguinotomía exploradora con biopsia peroperatoria de teste izquierdo cuyo resultado anatomopatológico fue de seminoma clásico con áreas de tumor del saco vitelino que infiltraba focalmente la túnica albuginia sin sobrepasarla. Este diagnóstico se confirmó posteriormente por el estudio inmunohistoquímico (Fig. 2).

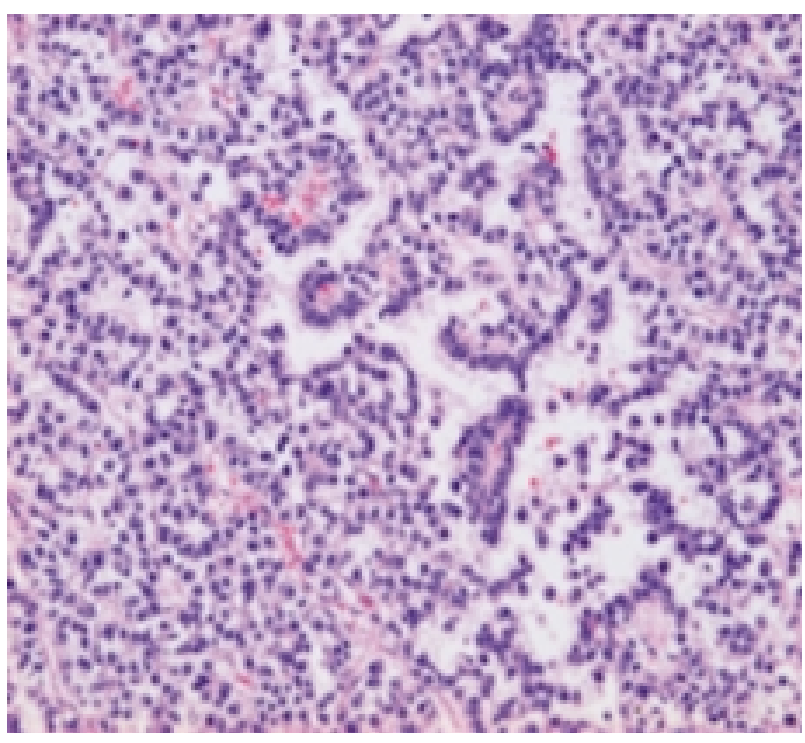

Figura 2. Histologia: seminoma clásico con zonas de tumor del saco vitelino. Diagnóstico confirmado por inmunohistoquimica.

Tras practicar la orquiectomía inguinal izquierda y tratándose de un tumor en estadío $\mathrm{T}_{1} \mathrm{~N}_{0} \mathrm{M}_{0}$ se decide tratamiento conservador y controles periódicos cada 3 meses con exploración física, determinación de marcadores tumorales, TC y radiografía de tórax.

En uno de estos controles y transcurridos 11 meses desde la cirugía, el paciente refiere molestias testiculares de reciente aparición similares a las que motivaron su consulta inicial. En la ecografia se visualizaron 3 lesiones nodulares hipoecoicas de 16, 13 y $6 \mathrm{~mm}$ de diámetro dependientes del polo superior del teste derecho (Figs. 3 y 4 ).

Se orientó el caso como una segunda neoplasia testicular con carácter multifocal y se descartó cualquier tipo de tratamiento conservador sobre 


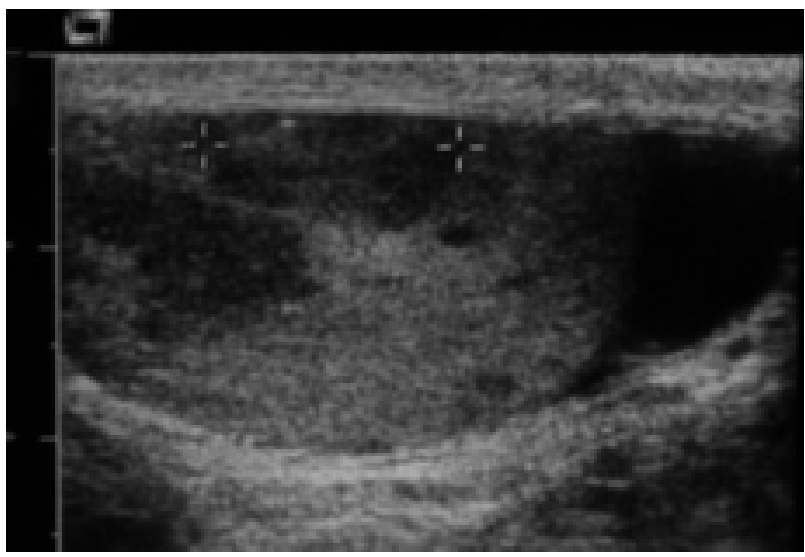

Figura 3. 2 de las 3 lesiones sólidas dependientes del polo superior del testiculo derecho.

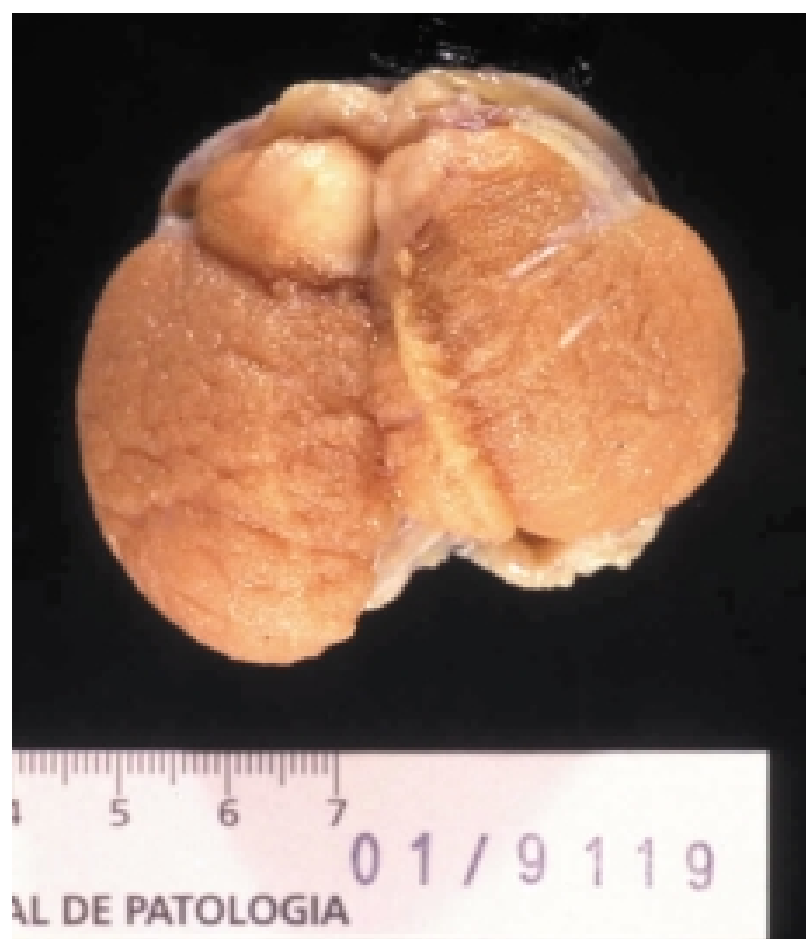

Figura 4. Sección sagital de testiculo derecho. Lesión nodular relativamente bien delimitada y de consistencia aumentada.

el testículo, practicándose nueva orquiectomía radical. El paciente rechazó la criopreservación de semen.

El estudio histológico confirmó la presencia de un seminoma clásico con zonas de CIS peritumorales sin invasión de la albuginia. Se trataba de nuevo de un tumor germinal estadío $\mathrm{T}_{1} \mathrm{~N}_{0} \mathrm{M}_{0}$.

El paciente sigue en la actualidad controles habituales cada 3 meses tras haber iniciado tera- pia hormonal sustitutiva. 14 meses después el paciente se encuentra asintomático y sin evidencia de enfermedad tumoral.

\section{DISCUSIÓN}

Uno de los principales factores de riesgo para presentar una neoplasia testicular es haber tenido un primer tumor en el teste contralateral. Un TGT previo aumenta la probabilidad de desarrollar una segunda neoplasia contralateral en un $24,8 \%$, representando un incremento del riesgo relativo de entre 19,4 y 35,7 veces respecto a la población general según las diversas series ${ }^{4}$. En el trabajo publicado por Coogan (1991) en el que se describen 2.088 pacientes diagnosticados de cáncer testicular, solamente el $24 \%$ de los tumores bilaterales se presentaron de forma sincrónica. El $76 \%$ restante de los casos fueron metacrónicos ${ }^{2}$.

La prevalencia de tumores bilaterales de testículo ha aumentado en los últimos tiempos. La mayor supervivencia de estos pacientes a raíz de la introducción de la quimioterapia con Cisplatino parece tener un papel relevante.

También se ha comunicado una incidencia relativamente mayor de tumores de teste en gemelos, hermanos y miembros de una misma familia pero no existen datos convincentes que indiquen una clara influencia genética. La incidencia de 1$4 \%$ de tumores bilaterales podría reflejar la importancia potencial de los factores genéticos o congénitos. Sin embargo no existen todavia evidencias científicas al respecto.

El tipo histológico del primer tumor no aumenta la probabilidad de desarrollar una segunda neoplasia y tampoco predice su histología. Sin embargo lo más común es observar el mismo tipo histológico en ambos testes, siendo el más frecuente el seminoma bilateral (en el $48 \%$ de los casos). En el $15 \%$ de casos serán tumores no seminomatosos de la misma histología; y se observan TGT de diferente tipo histológico en el 15\% de ocasiones, siendo éste el caso que presentamos.

El 22\% restante lo constituyen los tumores no germinativos ${ }^{5}$.

Alrededor del 7\% al 10\% de los pacientes con tumores de teste presentan antecedentes de criptorquidia y entre un 5\% y un $10 \%$ de ellos desarrollan una neoplasia en el teste contralateral normalmente descendido ${ }^{5}$. 
Berthelsen y col. (1982) encontraron la presencia de carcinoma "in situ" (CIS) en 13/250 $(5,2 \%)$ de los casos diagnosticados de cáncer en el teste contralateral, y de ellos un tercio presentaban atrofia testicular (tanto la urliana como la inespecífica) y una quinta parte de los pacientes tenían antecedentes de criptorquidia ${ }^{13}$.

La mayoría de autores coinciden en que el factor predictivo más potente es la presencia de CIS en la biopsia del teste contralateral. El CIS diagnosticado en varones post-puberales aparece como precursor de TGT "invasivo" en el 50\% de los casos a los 5 años. Aunque se ha descrito algún caso de pacientes portadores de CIS libres de tumor a los 10 años parece lógico pensar que terminarian desarrollando neoplasia si prolongáramos el tiempo de seguimiento.

Existe concordancia entre la prevalencia de CIS en pacientes menores de 30 años y la mayor prevalencia de TGT en este grupo de edad. Solamente el 6\% de los pacientes portadores de CIS son mayores de 45 años y no se ha detectado ningún caso de carcinoma in situ por encima de los 50 años $^{8,9}$.

Se desconoce el significado y comportamiento del CIS antes de la edad puberal. Skakkebaek (1987) propone que la progresión de CIS a TGT es un proceso hormonodependiente.

Por todo lo expuesto, parece prudente realizar una biopsia aleatoria durante la orquiectomía del teste contralateral en pacientes con factores de riesgo asociados, especialmente si coexisten 2 o más de los factores anteriormente mencionados. Obrando de este modo obtendremos mayor rentabilidad diagnóstica de la biopsia aunque dejaremos de detectar un 60\% de casos de CIS según apunta Dieckmann (1996) en su trabajo. Aunque una biopsia negativa no descarta la posibilidad de desarrollar un segundo tumor de testículo, disminuye el riesgo 50 veces en comparación con el resto de pacientes con un TGT no biopsiados ${ }^{9}$.

La necesidad de la biopsia testicular contralateral de forma rutinaria es un tema ampliamente debatido y sobre el cual no existe consenso en la actualidad. Sin embargo algunos autores de países centroeuropeos (principalmente germánicos) han optado por esta opción debido a que la prevalencia de CIS en estas zonas es del 5\%. En nuestro medio la prevalencia de CIS no supera el $2 \%$.
Como no se ha demostrado hasta la fecha la regresión espontánea de CIS y teniendo en cuenta que se trata de una lesión potencialmente letal la actitud del urólogo en caso de diagnosticarlo deberá ser sin duda con intención curativa, bien sea de forma inmediata o diferida en caso de que el paciente deseara descendencia en un breve periodo de tiempo.

Es importante tener en cuenta que la quimioterapia sistémica no asegura la erradicación del CIS; aunque éste desaparece con la quimioterapia en el 86\% de las ocasiones, el riesgo acumulado de recidiva se sitúa en el $21 \%$ a los 5 años y el $42 \%$ a los 10 años ${ }^{10}$. Este hecho puede explicarse por la existencia de la barrera hematotesticular.

Disponemos por tanto de dos únicas opciones terapéuticas: la orquiectomía radical y la radioterapia local sobre el teste en dosis fraccionadas menores de 20 Gy. A dosis de 18 y 16 Gy se obtienen buenas respuestas con preservación de las células de Leyding sin reducir la eficacia de la terapia.

Una alternativa a la orquiectomía radical en tumores testiculares bilaterales es la cirugía conservadora. Los grupos del Centro y Norte de Europa indican la cirugía parcial en TGT bilaterales o alojados en teste único siempre y cuando su tamaño sea menor o igual a los $25 \mathrm{~mm}$ y se trate de tumores organoconfinados. También se ha empleado la radioterapia local en caso de existir CIS peritumoral asociado.

La incidencia de recidiva local en el caso de tratamiento quirúrgico conservador es del $3 \%$. Por todo ello algunos autores recomiendan seguimiento estricto, incluyendo una biopsia testicular a los 6 meses de tratamiento ${ }^{11}$.

\section{REFERENCIAS}

1. ROMERO FERNÁNDEZ FJ, SÁENZ CUSÍ A, GIL SANZ MJ y cols.: Epidemiología de los tumores testiculares. Epidemiología descriptiva y analítica en Urología oncológica. Tema monográfico LXIII Congreso Nacional de Urología. ENE Ediciones 1998: 95-101.

2. COOGAN CL, FOSTER RS, SIMMONS GR y cols.: Bilateral testicular tumors: manegement and outcome in 21 patients. Cáncer 1998; 83: 547-552.

3. DIECKMANN KP, LOY V, BÜTTNER P y cols.: Prevalence of bilateral testicular germ cell tumors and early detection based on contralateral testicular intraepithelial neoplasia. Br J Urol 1993; 71: 340-345. 
4. OSTERLIND A, BERTHELSEN JG, ABILDGAARD N y cols.: Risk of bilateral testicular germ cell cancer in Denmark: 1960-1984. J Natl Cancer Inst 1991 oct. 2; 83: 1.391-1.395.

5. RICHIE JEROME P.: Neoplasias testiculares en: Campbell Urología sexta edición. Editorial Médica Panamericana 1994: 1.220-1.262.

6. SKAKKEBAEK NE, BERTHELSEN JG, GIWERCMAN A y cols.: Carcinoma in situ of the testis: possible origin from gonocytes and precursor of all types of germ cell tumours escept spermatocytoma. Int $J$ Androl 1987; 10: 19-28.

7. MUÑOZ VÉLEZ D, MUS MALLEU A, REBASSA LLULL M y cols.: Tumores germinales bilaterales de testículo. Aportación de cinco casos y revisión de la literatura. Actas Urol Esp 2000; 24: 632-639.

8. GIWERCMAN A, VON DER MAASE H, SKAKKEBAEK NE y cols.: Epidemiological and clinical aspects of carcinoma in situ of the testis. Eur Urol 1993; 23: 104-114.

9. DIECKMANN KP, LOY V.: Prevalence of contralateral testicular intraepithelial neoplasia in patients with testicular germ cell neoplasm. $J$ Clin Oncol 1996; 14: 3.126-3.132.

10. CHRISTENSEN TB, DAUGAARD G, GEERTSEN PF y cols.: Effect of chemotherapy on carcinoma in situ of the testis. Ann Oncol 1998; 9: 657-660.
11. FERNÁNDEZ APARICIO T, MIÑANA LÓPEZ B, CHAVES A y cols.: Tratamiento conservador del tumor testicular de células germinales. Actas Urol Esp 2000; 24: 810-816.

12. DIECKMANN KP, BOECKMANN W, BROSIG W y cols.: Bilateral testicular germ cell tumors. Report of 9 cases and review of the literature. Cancer 1998; 57: 1.254.

13. BERTHELSEN JG, SKAKKEBAEK NE.: Value of testicular biopsy in diagnosis carcinoma in situ of testis. Scand J Urol Nephrol 1981; 15: 165-168.

14. TEKIN A, AYGUN YC, AKI FT, OZEN H.: Bilateral germ cell cancer of the testis: a report of 11 patients with a long term follow-up. Br J Urol 2000; 85: 864868.

Dr. D. Cañís Sánchez

Servicio de Urología

Hospital del Mar

Passeig Maritim, 25-29

08003 Barcelona

(Trabajo recibido el 16 julio de 2002) 